\title{
Antibody response to a sterile filtered PPD tuberculin in M. bovis infected and M. bovis sensitized cattle
}

\author{
Bryan Rennie ${ }^{1,2^{*}}$, Lionel G Filion ${ }^{2^{*}}$, Nonie Smart ${ }^{1}$
}

\begin{abstract}
Background: Bovine tuberculosis, caused by Mycobacterium bovis, afflicts approximately 50 million cattle worldwide and is detected by the tuberculin skin test (TST). While it has long been recognized that purified protein derivative (PPD) tuberculin is composed of a mixture of $M$. bovis derived protein components, little is known about the quality, relative quantity and identity of the proteins that make up PPD tuberculin. We manufactured a sterile filtered PPD tuberculin (SF-PPD) from a nine-week-old M. bovis culture supernatant in order to characterise the culture filtrate proteins (CFP) which make up M. bovis PPD tuberculin and to compare the antibody response of M. bovis infected versus M. bovis sensitized cattle.

Results: SF-PPD resolved into approximately 200 discrete spots using two-dimensional polyacrylamide gel electrophoresis (2-DE) while fewer than 65 spots could be discerned from 2-DE gels of tuberculin derived from autoclaved culture supernatant. Two dimensional Western blot analyses indicated that sera from $M$. bovis sensitized cattle recognized additional SF-PPD antigens as compared to $M$. bovis infected cattle at seven weeks post infection/sensitization. However, application of a comparative tuberculin skin test resulted in an antibody boosting response to the same set of $M$. bovis CFPs in both the $M$. bovis infected and $M$. bovis sensitized cattle.

Conclusions: We concluded that it is the heat sterilization of the M. bovis CFPs that causes severe structural changes to the $M$. bovis proteins. This work suggests that $M$. bovis infected cattle and cattle artificially sensitized to $M$. bovis with an injection of heat killed cells exhibit similar antibody responses to M. bovis antigens.
\end{abstract}

\section{Background}

Bovine tuberculosis, caused by Mycobacterium bovis infection, is a major global health threat, with approximately 50 million cattle currently infected worldwide [1]. The primary method used to detect tuberculosis in cattle is the single intradermal test (SIT). Although the SIT is the most widely used diagnostic test for M. bovis infections in cattle, little is known about the quality, relative quantity and identity of the proteins that make up purified protein derivative (PPD) tuberculin.

PPD tuberculin is a crude and complex mixture of tuberculo-proteins which has changed little since its

\footnotetext{
* Correspondence: rennieb@inspection.gc.ca; Lionel.Filion@uOttawa.ca ${ }^{1}$ Canadian Food Inspection Agency, Mycobacterial Diseases, Ottawa, K2H 8P9, Canada

${ }^{2}$ Department of Biochemistry, Microbiology and Immunology, Faculty of Medicine, University of Ottawa, Ottawa, K1H 8M5, Canada

Full list of author information is available at the end of the article
}

conception and original application by Dr. Robert Koch in 1890 [2]. The original tuberculin, Koch's old tuberculin, was prepared from a heat sterilized liquid culture medium containing 8 - 12 week old $M$. tuberculosis $(M . t b)$ cultures concentrated to one-tenth the original volume by evaporation [2]. While it has long been recognised that PPD tuberculin is composed of $M$. bovis derived protein components, early efforts to accurately characterize the antigenic components of PPD tuberculin [3] were met with difficulty. In retrospect, interpretation of early findings were likely further complicated by protein denaturing effects of heat and pressure exerted during autoclaving and the absence of effective protein separation and characterization techniques. Fractionation of tuberculin into 3 - 14 antigenic fractions by alcohol fractionation $[4,5]$, column chromatography [6-8], and crossed immunoelectrophoresis [9] resulted in the description of a variety of tuberculo-protein fractions 
with incompletely defined structural characteristics and/ or biological activity such as the Antigen "L" [7] and PPD tuberculin fractions A, B and C [5].

With the advancement of molecular separation techniques and polyacrylamide gel electrophoresis (PAGE) examination of non-heated $M . t b$ [10] and $M$. bovis $[10,11]$ culture broths more than 800 tuberculo-proteins are currently described in the literature. Consequently, many of the tuberculin fractions previously described as homogenous entities actually consist of multiple mycobacterial proteins. The current accepted terminology for this complex mixture of tuberculo-proteins is culture filtrate proteins (CFP) and this includes secreted proteins, exported proteins and non-secreted, somatic components which are released into the culture medium due to autolysis, replication and bacterial leakage [12,13]. The protein profile of a CFP set is dependent on many factors including cultivation time, temperature, growth medium and culture agitation $[13,14]$.

Today most laboratories use non-heated $M$. bovis culture filtrates rather than tuberculin for the identification of specific $M$. bovis antigens for use as diagnostic and vaccine candidates $[10,12,13,15-17]$. Separation and characterization of non-heated $M$. bovis CFPs using molecular techniques such as two dimensional polyacrylamide gel electrophoresis (2-DE), mass spectronomy (MASS-SPEC) analysis and in vitro antigenicity assays has lead to the identification of several, highly antigenic $M$. bovis proteins. However, the antigenic activity of these proteins and their conservation in field-use $M$. bovis PPD tuberculin remains largely unknown.

Analysis of $M$. bovis culture filtrate proteins with two dimensional polyacrylamide gel electrophoresis (2-DE) has indicated that PPD tuberculin is derived from a multitude of tuberculo-proteins [10-13]. Tuberculin manufacturing methods, which include heating and chemical treatment, may alter the structure and antigenicity of the tuberculo-proteins in field issue PPD tuberculin. In accordance with international standards [18] the antigenicity of each new batch of PPD tuberculin, in many laboratories, is routinely measured in animals sensitized with heat killed $M$. bovis cells.

As many of the antigenic $M$ bovis CFP have been shown to elicit both cellular and humoral responses in cattle $[1,19,20]$, we hypothesized that immunological differences would be observed between cattle infected with $M$. bovis as compared to cattle artificially sensitized to $M$. bovis with an injection of heat killed cells. Our experiments presented in this manuscript showed that classical, autoclaved PPD was essentially a mixture of peptide fragments which could not be sufficiently resolved by SDS-PAGE, 2-DE or Western blot analysis to characterize the antibody responses of our experimental cattle. $M$ bovis sterile culture filtrate from 9-week-old cultures, however, could be satisfactorily resolved by these same methods. We concluded that it was the heat sterilization of the M. bovis CFPs that caused the severe structural changes and protein fragmentation to the $M$. bovis proteins. The immunological significance of the protein fragmentation, however, remains to be determined. This work also suggested that $M$. bovis infected cattle and cattle artificially sensitized to $M$. bovis with an injection of heat-killed cells possess similar antibody response to selected $M$. bovis antigens. While this study compares the humoral immune response of $M$. bovis infected and $M$. bovis sensitized cattle, future comparisons of their cellular immune responses may lead an $M$. bovis sensitization method which would provide a consistent immune response similar to that of an experimentally $M$. bovis infected animals. The development of an accurate, noninfectious bovine tuberculosis model would reduce the complexity and bio-containment risks associated with cattle studies involving live $M$. bovis.

\section{Methods}

\section{Production of $M$. bovis SF-PPD and PPD tuberculin}

M. bovis AN5 was cultured aerobically at $37 \pm 2{ }^{\circ} \mathrm{C}$ on Reid's synthetic, liquid medium. After nine weeks of incubation, the culture flasks were divided into two groups. Culture supernatant from the first group was inactivated by autoclaving at $121^{\circ} \mathrm{C}$ and $110 \mathrm{kPa}$ for $45 \mathrm{~min}$. Tuberculin produced from this culture supernatant was referred to as heat killed PPD tuberculin (HK-PPD). Culture supernatant from the second group was separated from the live $M$. bovis cells by filtration through a $3 \mu \mathrm{m}$ and a $0.8 / 0.2$ $\mu \mathrm{m}$ capsule filters (Pall Corporation, USA) arranged in series. Tuberculin produced from this culture supernatant was referred to as sterile filtered PPD tuberculin (SF-PPD). Both the sterile filtered and autoclaved culture supernatant was confirmed sterile by culture.

Both the sterile filtered and autoclaved culture supernatants were filtered at $0.22 \mu \mathrm{m}$ and concentrated with $10 \mathrm{kDa}$ tangential flow filtration (TFF) cassettes (Pellicon Cassette, Millipore Corporation, USA). Tuberculoproteins were precipitated by addition of ammonium sulfate, pelleted by centrifugation, re-suspended in a phenolized phosphate buffer $(0.0147 \%(\mathrm{w} / \mathrm{v})$ sodium phosphate dibasic, $0.076 \%(\mathrm{w} / \mathrm{v})$ potassium phosphate, $0.5 \%(\mathrm{v} / \mathrm{v})$ phenol) and de-salted/concentrated with 10 $\mathrm{kDa}$ TFF. Further dialysis to remove buffer salts and phenol from both SF-PPD and HK-PPD was accomplished using a 5-kDa TFF capsule filter (Minimate ${ }^{\mathrm{TM}}$ TFF System, Pall Corporation, USA).

Protein concentrations were determined using the $D C^{\mathrm{m} m}$ protein assay following the manufacturer's instructions with bovine serum albumin as standard (Bio-Rad, Mississauga, Ontario) [21]. 
PAGE and MASS SPEC analysis of HK-PPD and SF-PPD

One dimensional, vertical, sodium dodecyl sulphate (SDS) PAGE was performed as per Lamelli, with the following specifications [22]. A 6\% acrylamide, $\mathrm{pH} 6.8$ stacking gel and a $12 \%$ acrylamide, non-linear gradient, separation gel with a $\mathrm{pH}$ of 8.8 were used. The acrylamide-bis ratio was 29:1 and gels were cast in either $16 \times$ $20 \mathrm{~cm}$ or $8.3 \times 7.3 \mathrm{~cm}$ sizes with thicknesses of either $0.75,1.00$ or $1.50 \mathrm{~mm}$ depending on the application. Electrophoresis chemicals and molecular weight standards were purchased from Bio-Rad Laboratories, Mississauga, Ontario. HK-PPD and SF-PPD samples were placed 1:4 into a reducing sample buffer which contained 2.0\% (w/v) SDS, 5.0\% (vol/vol) 2-mercaptoethanol, $10 \%(\mathrm{v} / \mathrm{v})$ glycerol, $0.0625 \mathrm{M}$ tris base ( $\mathrm{pH}$ 6.8), and $0.005 \%(\mathrm{w} / \mathrm{v})$ bromophenol blue. Samples were boiled at $100^{\circ} \mathrm{C}$ for 5 minutes and centrifuged for 5 minutes at $14000 \mathrm{xg}$ prior to loading. The amount of protein applied to each lane varied with the application and size of the gel. Electrophoresis was performed using either the PROTEAN ${ }^{\odot}$ II or mini-PROTEAN ${ }^{\bullet}$ III cells (Bio-Rad) at $20 \mathrm{~mA}$ constant current per gel. Electrophoresis was stopped when the bromophenol blue tracking dye reached the bottom of the separating gel.

HK-PPD and SF-PPD samples separated by 2-DE were first subjected to iso-electric focusing (IEF) along an acrylamide strip, followed by SDS-PAGE, molecular weight separation in a direction 90 degrees from the IEF. The precast, $17 \mathrm{~cm}$ IEF strips had an immobilized $\mathrm{pH}$ range of 3-10 (ReadyStrip ${ }^{\mathrm{Tx}}$; Bio-Rad Laboratories). Isoelectric focusing was carried out using a Multiphor ${ }^{\mathrm{TM}}$ II Electrophoresis Unit (Amersham Pharmacia Biotech) and a PowerPac ${ }^{\mathrm{TM}} \mathrm{HV}$ power supply (Bio-Rad Laboratories, Mississauga, Ontario). IEF strips were re-hydrated and loaded with $300 \mu \mathrm{L}$ of either HK-PPD or SF-PPD prepared in ReadyPrep ${ }^{\mathrm{m}}$ Squential Extraction Kit Reagent 3 (Bio-Rad Laboratories) with $3 \mu \mathrm{L}$ of tributyl phosphine and $0.0003 \%(\mathrm{w} / \mathrm{v})$ bromophenol blue. PPD samples were diluted in de-ionized water according to application and strips were focussed for $\sim 98,000$ volt hours.

Prior to the second dimension, IEF strips were equilibrated sequentially for $15 \mathrm{~min}$. at $37^{\circ} \mathrm{C}$ in first a reduction buffer $(0.05 \mathrm{M}$ Tris, $\mathrm{pH}$ 6.8; $8 \mathrm{M}$ urea; $35 \%$ glycerol; $2 \%$ $(\mathrm{w} / \mathrm{v})$ dithiothreitol; $0.3 \%$ SDS) followed by an alkylation buffer (0.05 M Tris, pH 6.8; $8 \mathrm{M}$ urea; 35\% glycerol; $2.5 \%$ (w/v) iodoacetamide; $0.3 \%$ SDS). Following alkylation, the IEF strips were loaded into $12 \%$ SDS polyacrylamide gels with the $\mathrm{pH} 3$ end of the strip towards the molecular weight marker and overlaid with $0.5 \%(\mathrm{w} / \mathrm{v})$ low melting agarose containing $0.0001 \%(\mathrm{w} / \mathrm{v})$ bromophenol blue. Second dimension electrophoresis was performed using the PROTEAN ${ }^{\circledR}$ II cell (Bio-Rad) at 20 mA constant current per gel. Electrophoresis was stopped when the bromophenol blue tracking dye reached the bottom of the separating gel. Electrophoresis chemicals and molecular weight standards were purchased from BIO-RAD Laboratories, Mississauga, Ontario.

Gels were stained with either Comassie Brilliant Blue (CBB) R-250 stain (Bio-Rad Laboratories, Mississauga, Ontario) or with a non-fixing silver stain method described by Shevchenko et al. (1996) [23].

SDS-PAGE and 2DE gels of HK-PPD and SF-PPD were digitized in 8 bit greyscale at 300 dpi with a ScanMaker $i 900$ scanner (Microtek). Spots on the 2DE gels were enumerated by PDQuest 7.1.0 2D Analysis Software (Bio-Rad). Following automated enumeration, erroneous and duplicate spots were manually deleted.

Nine spots of interest were excised from a CBB stained 2DE gel of SF-PPD $(200 \mu \mathrm{g} ; 50 \mu \mathrm{L}$ of $4 \mu \mathrm{g} / \mu \mathrm{L})$. Excised spots were placed in sterile vials and maintained at $-80^{\circ} \mathrm{C}$ prior to submission to the Ottawa Institute for Systems Biology (University of Ottawa) for MASS-SPEC analysis.

\section{Western blot analysis of $M$. bovis Infected and $M$. bovis sensitized cattle sera}

The cattle sera used for Western blots were collected from twenty-four Holstein cross cattle in 2004 during a comparative intradermal tuberculin skin test (CITST) study. CITST methodology was based on recommendations from the World Organization for Animal Health's Manual of Diagnostic Tests and Vaccines for Terrestrial Animals [18]. In this study twelve cattle received a $1.0 \mathrm{~mL}$ intra-tracheal inoculation of $1500 \mathrm{CFU}$ of virulent $M$. bovis (field strain 02/1007; CFIA designation) and another twelve cattle received a $1 \mathrm{~mL}$ intramuscular injection containing $20 \mathrm{mg}$ of heat killed (autoclaved) M. bovis cells (field strain 02/1007, CFIA designation) suspended in 50\% mineral oil, 25\% lanolin and $25 \%$ saline. One negative control animal (cattle \# 9246) received a $1 \mathrm{~mL}$ injection containing 50\% mineral oil, 25\% lanolin and $25 \%$ saline. $M$. bovis sensitized cattle serum from a previous experiment was used as a positive control (cattle \# 893). Cattle were screened with an in vitro blood based assay (Bovigam ${ }^{\mathrm{Tm}}$; Pfizer, Australia) prior to infection/sensitization and based on the manufacturer's cutoff values were determined to be negative for bovine tuberculosis. One-dimensional Western blot analyses were performed on sera derived from three $M$. bovis sensitized cattle (cattle \# 003,457, 993) and two M. bovis infected cattle (cattle \# 103, 107). Two-dimensional Western blot analyses were performed on sera derived from six $M$. bovis sensitized cattle (cattle \# 003, 112, 207, 211, 457, 993) and six M. bovis infected cattle (cattle \# 103, 106, 107, 108, 109, 110).

Blood was collected weekly for a period of twenty weeks from all cattle and the sera was maintained at $-80^{\circ} \mathrm{C}$. 
CITSTs were applied to both the sensitized and infected cattle at 7 and 13 weeks post sensitization/infection respectively. The Ottawa Laboratory Fallowfield CFIA Institutional Animal care committee approved all animal use and procedures in these studies. All animals received ad-libitum food and water and were handled and cared for in accordance with the regulations prescribed by the Canadian Council on Animal Care. M. bovis infection status was confirmed in all cattle inoculated with live M. bovis by lesions observed on necropsy, by histological identification of acid-fast bacteria with typical histopathology of mycobacteriosis and by isolation of $M$. bovis on culture. Likewise, all artificially sensitized cattle were confirmed negative for $M$. bovis infection by the same tests.

Western immunoblotting of $M$. bovis sensitized/ experimentally infected cattle sera onto either HK-PPD or SF-PPD was performed as per Towbin et al, with the following specifications [24]. Electrophoretically separated HK-PPD and SF-PPD was transferred onto nitrocellulose membranes $(0.45 \mu \mathrm{m}$ pore size, Bio-Rad Laboratories) using a tank style blotting system (Transphor Electrophoresis unit, Hoefer Inc., USA) at 100 volts for 1 hour and blocked for 1 hour at $37^{\circ} \mathrm{C}$ in 7.4 $\mathrm{pH}$, Tris buffered saline (TBST) $(0.02 \mathrm{M}$ Tris, $0.8 \%$ $(\mathrm{w} / \mathrm{v})$ sodium chloride, $0.02 \%(\mathrm{w} / \mathrm{v})$ potassium chloride, 0.3 (v/v) Tween 20). The nitrocellulose was incubated overnight at room temperature in either $M$. bovis sensitized or infected bovine sera diluted 1:200 in TBST. Nitrocellulose was washed with TBST, incubated for 2 hours at room temperature with alkaline phosphatase conjugated rabbit anti-bovine IgG (Sigma-Aldrich Canada Ltd., Oakville, Ontario) diluted 1:5000 in TBST. Phosphatase substrate (5-bromo-4-chloro-3-indolylphosphate/nitroblue tetrazolium, (Mandel Scientific Company Inc., Guelph, Ontario) was applied to the nitrocellulose for 10-15 minutes at which time the reaction was stopped with the addition of de-ionized water. Western blots were examined visually and were also digitized in 8-bit greyscale at $300 \mathrm{dpi}$ with a ScanMaker i900 scanner (Microtek).

\section{Results}

HK-PPD and SF-PPD examination by SDS-PAGE

The components of HK-PPD and SF-PPD were separated using SDS-PAGE and stained with either Coomassie Brilliant Blue $(\mathrm{CBB})$ or silver stain so that the denaturing effect of autoclaving on $M$. bovis culture filtrate proteins could be visualized. Electrophoresis of HK-PPD resulted in the appearance of two blurred bands of approximately 10 and $23 \mathrm{kDa}$ (Figure 1, lane 4). The profile of the HKPPD was also dominated by the appearance of a large streak that commenced at approximately $45 \mathrm{kDa}$ and increased in intensity at less than $25 \mathrm{kDa}$.

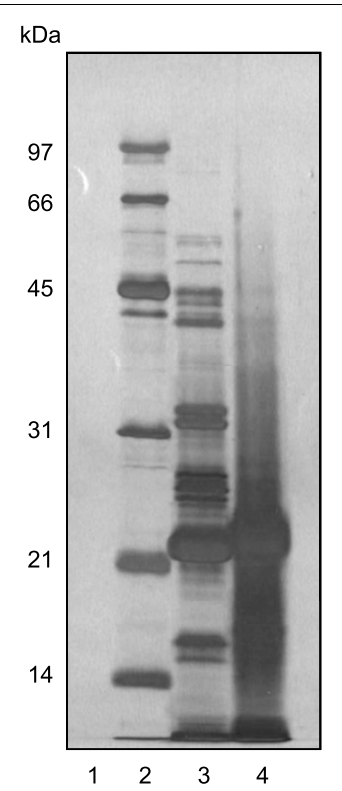

Figure 1 Twelve percent SDS-PAGE analysis of HK PPD and SF-PPD. (a) Gel stained with non-fixing silver stain. Lanes: 1, Sample buffer; $50 \mu \mathrm{L}$. 2. molecular weight standards (Silver Stain SDS-PAGE Standards Low Range, BIO-RAD). 3, SF-PPD; 12.5 g. 4, HK-PPD; 12.5 ug.

In comparison to HK-PPD, at least 35 bands could be visualized in SDS-PAGE of SF-PPD, many of which ranged from 23 to $80 \mathrm{kDa}$ (Figure 1, lane 3 ). The two bands noted in PAGE of HK-PPD had corresponding bands of similar molecular weight in PAGE of SF-PPD however; the corresponding SF-PPD bands were discrete. Furthermore, the profile of SF-PPD lacked the streaked appearance noted in HK-PPD lanes.

\section{Western immunoblot comparison of HK-PPD and SF-PPD}

The protein denaturing effect of autoclaving on M. bovis CFPs shown by PAGE and 2DE comparisons of HK-PPD versus SF-PPD tuberculin is suggested that the antigenicity of the SF-PPD differed from that of HK-PPD. Therefore, in order to investigate this further, SF-PPD and HK-PPD were compared by Western blot analysis using serum from $M$. bovis sensitized and infected cattle. Western blots of HK-PPD with either $M$. bovis infected or $M$. bovis sensitized sera revealed that the majority of antibody binding was restricted to one, predominant, poorly delineated $22 \mathrm{kDa}$ band and to two additional faint bands of approximately 40 and $66 \mathrm{kDa}$ (Figure 2, lane 6, 7). Contrary to SDS-PAGE analysis (Figure 1, lane 4), Western blots of HK-PPD did not detect antigen less than $20 \mathrm{kDa}$.

In comparison, Western blots of SF-PPD using the same $M$. bovis sensitized and $M$. bovis infected cattle sera, recognized more than 10 bands with molecular weights ranging from 16 - $90 \mathrm{kDa}$ (Figure 2, lane 4, 5). 
As with blots of HK-PPD, few bands were detected at less than $20 \mathrm{kDa}$ by either the $M$. bovis sensitized or infected sera, however, the sera did recognise several SF-PPD bands greater than $20 \mathrm{kDa}$ which corresponded with bands observed in silver stained PAGE of SF-PPD (Figure 1). Small variations were observed in the Western blot banding patterns of SF-PPD among $M$. bovis infected and $M$. bovis sensitized sera. However, no consistent difference was observed in the Western blot banding patterns of SF-PPD probed with sera from the two groups of cattle, small variations were also observed among blots probed with sera obtained from individual cattle within both the M. bovis sensitized, and the $M$. bovis infected groups (data not shown).

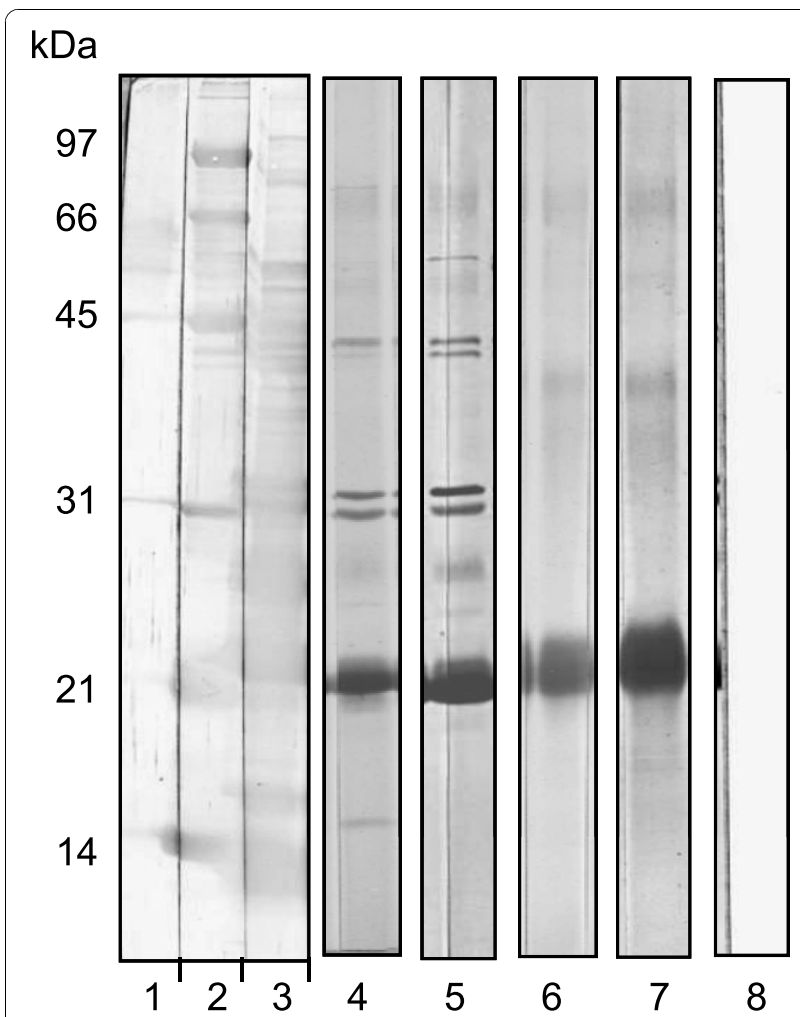

Figure 2 Western blot analysis of HK-PPD and SF-PPD probed with either $M$. bovis infected or $M$. bovis sensitized cattle sera. Twelve percent SDS-PAGE were performed followed by electrophoretic transfer of proteins to nitrocellulose. Lanes were cut into strips and individually stained or blotted. (a) Lanes 1, 2 and 3 were loaded with sample buffer, molecular weight standards (Silver Stain SDS-PAGE Standards Low Range, Bio-Rad) and $10 \mu \mathrm{g}$ of SF-PPD respectively and were stained with Colloidal Gold (Bio-Rad) following transfer. Lanes 4 and 5 were loaded with $10 \mu \mathrm{g}$ of SF-PPD and lanes 6 and 7 were loaded with $10 \Phi \mathrm{g}$ of HK-PPD. Lanes 4 and 6 were blotted with $M$. bovis sensitized bovine sera (animal \# 003); lanes 5 and 7 were blotted with $M$. bovis infected bovine sera (animal \# 107). Lane 8 was loaded with $10 \mu \mathrm{g}$ of HK-PPD and was blotted without sera $\left(1^{\circ} \mathrm{Ab}\right)$ to act as a conjugate control.

\section{2-DE analysis of SF-PPD and HK-PPD}

Although SDS-PAGE of SF-PPD resulted in improved delineation of proteins as compared to HK-PPD, it was hypothesized that two-dimensional SDS-PAGE (2-DE) would offer additional protein separation. Approximately 196 spots were enumerated from digitized 2DE images of SF-PPD by PDQuest 2D Analysis Software (Bio-Rad), the majority of which localized within a pI range of 4 6 and within a molecular weight range less than $80 \mathrm{kDa}$ (Figure 3a).

In comparison to SF-PPD, 2D gels of HK-PPD were dominated by an indistinct smear which had a pI range of $3-5$ and a molecular weight range of $0-80 \mathrm{kDa}$ (Figure $3 \mathrm{~b}$ ). The majority of the HK-PPD components had molecular weights less than $25 \mathrm{kDa}$, and were blurred as compared to those of the SF-PPD gels. HKPPD 2-DE gels also exposed approximately 30 spots from $5-15 \mathrm{kDa}$, in the $5.5-7.5 \mathrm{pI}$ range that were not visualized in SF-PPD gels. A reduction from $50 \mu \mathrm{g}$ to 10 $\mu \mathrm{g}$ of the HK-PPD protein level applied to 2-DE reduced the intensity of the smear and facilitated the detection of additional faint spots. However, only 69 individual spots were enumerated by PDQuest in 2-DE of HK-PPD (Figure $3 \mathrm{~b}$ ) as compared to the 196 individual spots enumerated in SF-PPD (Figure 3a).

\section{MASS SPEC analysis of nine SF-PPD spots}

Nine 2DE SF-PPD spots were selected for MASS-SPEC analysis (Figure 3a). While the MASS-SPEC analysis potentially identified several mycobacterial proteins coexisting at each spot (TABLE 1), the SF-PPD spots were annotated based on the strength of association provided by the MASS-SPEC analysis (Mascot score) and by comparison to previously published 2-DE analysis of Mycobacterium spp. CFPs $[10,12,15,16,25,26]$. MPB32 was also annotated in Figure 3 a based on its identical position and appearance to previously published $2 \mathrm{DE}$ analyses of mycobacterial CFPs $[13,17,27]$.

\section{2-DE Western blot analysis of SF-PPD}

Following one-dimensional Western blot analyses on sera from both the $M$. bovis infected and M. bovis sensitized cattle groups, it became apparent that a twodimensional (2D) Western blot approach would offer an improved visualization of the antibody response to SF-PPD antigens. The majority of the antibody response was observed with components located between $4-6 \mathrm{pI}$ and within a molecular weight range of $20-36 \mathrm{kDa}$ (Figure 4). In comparison to 2-DE of SF-PPD (Figure $3 \mathrm{a}$ ), a series of spots at approximately $20 \mathrm{kDa}$ and another cluster of spots at approximately $30 \mathrm{kDa}$ appeared to be associated with the MPB70/MPB83 and Ag85 proteins detected by MASS-SPEC from 2-DE gels of SF-PPD (Figure 3a. A series of four spots at 


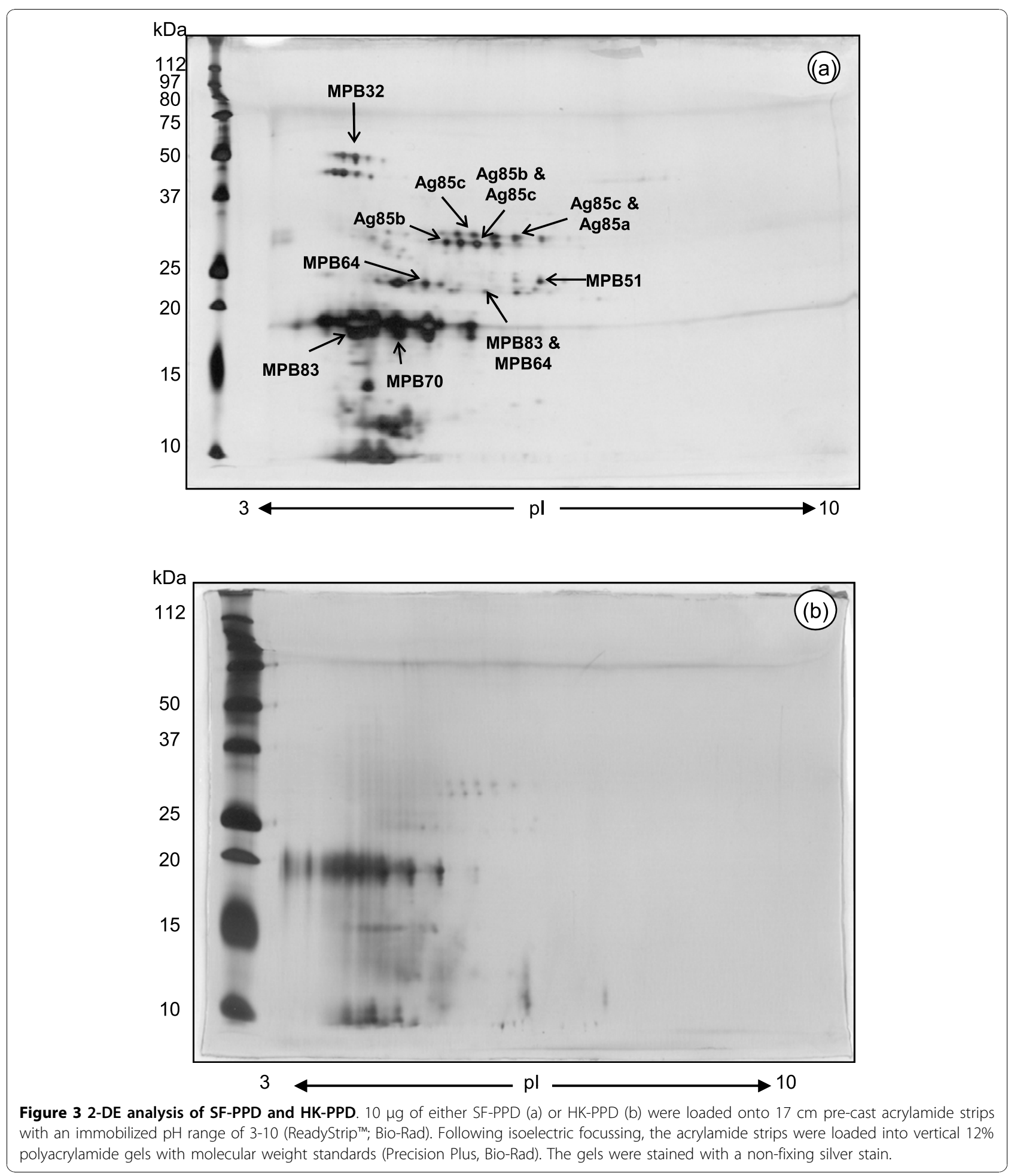

approximately $28 \mathrm{kDa}$ with an approximate pI range of 4.5 - 5.5 was also associated with MPB64 and MPB83 by the same analysis. Antibody response was also detected within a $40-80 \mathrm{kDa}$ and 4-8 $\mathrm{pI}$ range in the 2D Western blots. While the 40 - $80 \mathrm{kDa}$ spots do not pair up with spots observed in $10 \mu \mathrm{g}, 2$-DE gels of SF-PPD (Figure 3a), they can be associated with spots observed on 2-DE gels loaded with $50 \mu \mathrm{g}$ of SF-PPD (data not shown). In contrast to the 2-DE gels of SF-PPD, limited antibody response was observed to 
Table 1 Results of MASS-SPEC analysis of 9 spots excised from 2DE gel of SF-PPD

\begin{tabular}{|c|c|c|c|c|c|}
\hline \multicolumn{3}{|c|}{ SF-PPD spots for MASS SPEC analysis } & \multirow{2}{*}{\multicolumn{3}{|c|}{$\begin{array}{c}\text { Mascot Search Results } \\
\text { Protein hits }\end{array}$}} \\
\hline \multirow[b]{2}{*}{ Designation } & \multicolumn{2}{|c|}{$\sim$ 2DE spot location } & & & \\
\hline & $\operatorname{mass}(\mathrm{kDa})$ & $\mathrm{pl}$ & Name & $\operatorname{mass}(\mathrm{kDa})$ & Score \\
\hline \multirow[t]{5}{*}{ MPB83 } & 22 & 4.2 & MPB83 & 22.2 & 471 \\
\hline & & & CFP10 & 10.8 & 458 \\
\hline & & & MPB70 & 22.5 & 433 \\
\hline & & & MPB63 & 13.7 & 430 \\
\hline & & & ESAT-6 & 10.0 & 337 \\
\hline \multirow[t]{5}{*}{ MPB70 } & 22 & 4.7 & MPB70 & 22.5 & 588 \\
\hline & & & MPB83 & 22.2 & 473 \\
\hline & & & Rv1314c & 20.8 & 196 \\
\hline & & & CFP10 & 10.8 & 177 \\
\hline & & & MPB63 & 16.5 & 47 \\
\hline \multirow[t]{5}{*}{ MPB64 } & 25 & 4.5 & MPB64 & 25.1 & 733 \\
\hline & & & MPB83 & 22.2 & 509 \\
\hline & & & MPB70 & 22.5 & 434 \\
\hline & & & M.tb protease & 23.5 & 381 \\
\hline & & & lipoprotein LPPX & 24.3 & 377 \\
\hline \multirow[t]{5}{*}{ MPB83 \& MPB64 } & 25 & 5.4 & Adenylate kinase & 20.1 & 504 \\
\hline & & & MPB83 & 22.2 & 466 \\
\hline & & & MPB64 & 25.1 & 366 \\
\hline & & & Rv2557 & 24.7 & 356 \\
\hline & & & Ag85b & 30.8 & 339 \\
\hline \multirow[t]{5}{*}{ MPB51 } & 27 & 6 & MPB51 & 31.1 & 699 \\
\hline & & & enoyl-CoA hydratase & 24.5 & 506 \\
\hline & & & MPB64 & 25.1 & 145 \\
\hline & & & MPB70 & 22.5 & 122 \\
\hline & & & Peptide of a $24 \mathrm{kDa}$ immunoprotective protein & 2.1 & 99 \\
\hline \multirow[t]{5}{*}{ Ag85b } & 31 & 5 & Ag85b & 30.8 & 609 \\
\hline & & & M.tb protein MT3693 & 28.2 & 534 \\
\hline & & & Beta-1, glucanase precursor & 32.2 & 329 \\
\hline & & & chaperonin GroEL & 31.2 & 289 \\
\hline & & & 29 kDa Ag & 28.5 & 210 \\
\hline \multirow[t]{5}{*}{ Ag85b \& Ag85c } & 31 & 5.3 & Ag85b & 30.8 & 610 \\
\hline & & & $\mathrm{Ag} 85 \mathrm{C}$ & 31.2 & 417 \\
\hline & & & Ag85a & 32.7 & 276 \\
\hline & & & Conserved membrane protein & 27.3 & 107 \\
\hline & & & dehydrogenase/reductase & 29.9 & 42 \\
\hline \multirow[t]{4}{*}{$\mathrm{Ag} 85 \mathrm{C}$} & 32 & 5.3 & $\mathrm{Ag} 85 \mathrm{C}$ & 33.1 & 632 \\
\hline & & & esterase & 34.0 & 611 \\
\hline & & & Ag85b & 30.8 & 289 \\
\hline & & & Ag85a & 32.8 & 191 \\
\hline \multirow[t]{5}{*}{$\mathrm{Ag} 85 \mathrm{c} \& \mathrm{Ag} 85 \mathrm{a}$} & 32 & 5.7 & Ag85c & 33.1 & 578 \\
\hline & & & Ag85a & 32.8 & 351 \\
\hline & & & Ag85b & 30.8 & 309 \\
\hline & & & amidohydrolase & 29.1 & 181 \\
\hline & & & TB15.3 & 15.3 & 133 \\
\hline
\end{tabular}




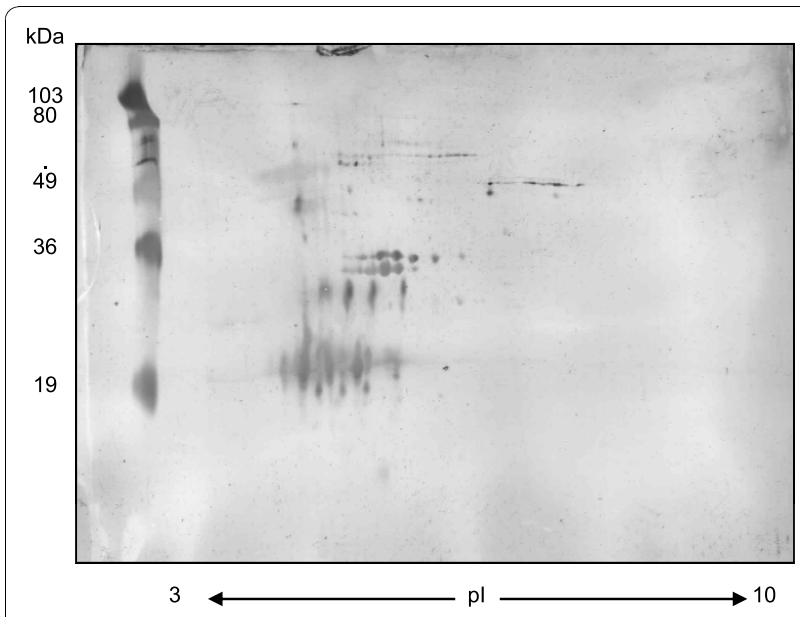

Figure 4 2D-Western blot analysis of the antibody response to SF-PPD proteins in sera from an $M$. bovis sensitized bovine. SF-PPD $(200 \mu \mathrm{g})$ was loaded onto a $17 \mathrm{~cm}$ pre-cast acrylamide strip with an immobilized $\mathrm{pH}$ range of 3-10 (ReadyStrip ${ }^{\mathrm{TM}}$; Bio-Rad). Following isoelectric focussing, the acrylamide strip was loaded into vertical $12 \%$ polyacrylamide gels with molecular weight standards (Pre-stained - Low range, Bio-Rad). Protein was electrophoretically transferred to nitrocellulose and blotted with $\mathrm{M}$. bovis sensitized cattle sera (animal \# 893).

components at less than $20 \mathrm{kDa}$ in $2 \mathrm{D}$ Western blots of SF-PPD.

Previous work in our laboratory showed that cattle artificially sensitized to $M$. bovis with an injection of heat killed M. bovis cells mounted a $30 \%$ greater average delayed type hypersensitivity (DTH) response to the intradermal application of $M$. bovis PPD tuberculin as compared to cattle experimentally infected with $M$. bovis (unpublished data). We hypothesized that immunological differences between these respective cattle models would be observed.

The serological response of six M. bovis infected and six $M$. bovis sensitized cattle were examined by 2D Western blot analysis (Figure 5). Sera from each animal were blotted at three time-points: pre-infection/pre-sensitization, seven weeks and thirteen weeks post sensitization or post infection (respectively) and three weeks post CITST. Every animal's pre-infection or pre-sensitization sera generated a background antibody response to SF-PPD antigens. While the background responses varied from animal to animal, the putative Ag85 complex was consistently observed with each animal's pre-infection or pre-sensitization sera (Figure 5a, b). A trend observed from the seven-week post infection/sensitization time-point indicated that the M. bovis sensitized cattle demonstrated a more intense antibody response and to additional M. bovis Ags, especially MPB70, MPB64 and MPB64/MPB83, as compared to the M. bovis infected cattle (Figure 5c, d, Additional files 1,
2, 3, 4, 5 and 6, FIG. S1 to S6). An overall, antibodyboosting effect was observed in the post-CITST timepoint 2D Western blots of both the sensitized and infected cattle (Figure 5e, f). While the antibody response to SF-PPD protein spots at this time-point varied between individual animals within both the sensitized and infected cattle groups, each group appeared to respond similarly to the majority of $M$. bovis protein spots (Additional files 1, 2, 3, 4, 5 and 6, FIG. S1 to S6). No changes were observed from pre-injection to postCITST in 2D Western blots of sera from a negative control animal which received a mineral oil/lanoline/saline injection (Additional file 7 FIG. S7).

\section{Discussion}

Since the introduction of the tuberculin skin test (TST) by Koch in 1890 [2], tuberculin has been produced from heat-sterilized $M$. bovis culture filtrate and the TST remains the principal ante-mortem diagnostic test for bovine tuberculosis worldwide. Remarkably, the composition of tuberculin and the antigenicity of its components remain largely unknown even though it is now recognized that 2-4 week old, non-heated $M$. bovis culture filtrate is composed of more than 800 CFPs [12]. Previous attempts to separate the active components of tuberculin by a variety of fractionation [3-8] and electrophoretic techniques $[9,28]$ have led to equivocal results and difficulty in interpretation, most likely due to the observation that heat killing during tuberculin production leads to profound structural and possibly other uncharacterized changes to the mycobacterial CFPs.

In this study, the effect of autoclaving on mycobacterial CFPs is readily evidenced by our observation that there is at least a three-fold increase in additional spots that can be enumerated in 2-DE gels of non-heated SF-PPD as compared to HK-PPD. Furthermore, the differences observed between gels of SF-PPD and HK-PPD do not appear to be restricted to protein denaturation as both SF-PPD and HK-PPD proteins are also denatured by the SDS-PAGE protocol which uses a combination of heat, SDS and 2-ME treatment prior to electrophoresis. Therefore, the effects of heat and pressure due to the autoclave process are likely responsible for the creation of tuberculo-protein peptide fragments in the HK-PPD preparation which appear as streaks or smears on PAGE or 2DE gels. These results are consistent with previous attempts to characterize the constituents of tuberculin $[2,9]$ all of which professed difficulty delineating protein bands from autoclaved preparations of tuberculin.

Although the majority of HK-PPD proteins appeared blurred as compared to of SF-PPD in 2-DE gels, the delineation of more than 60 individual protein spots in 2-DE of HK-PPD (Figure 3b) suggested that PPD 


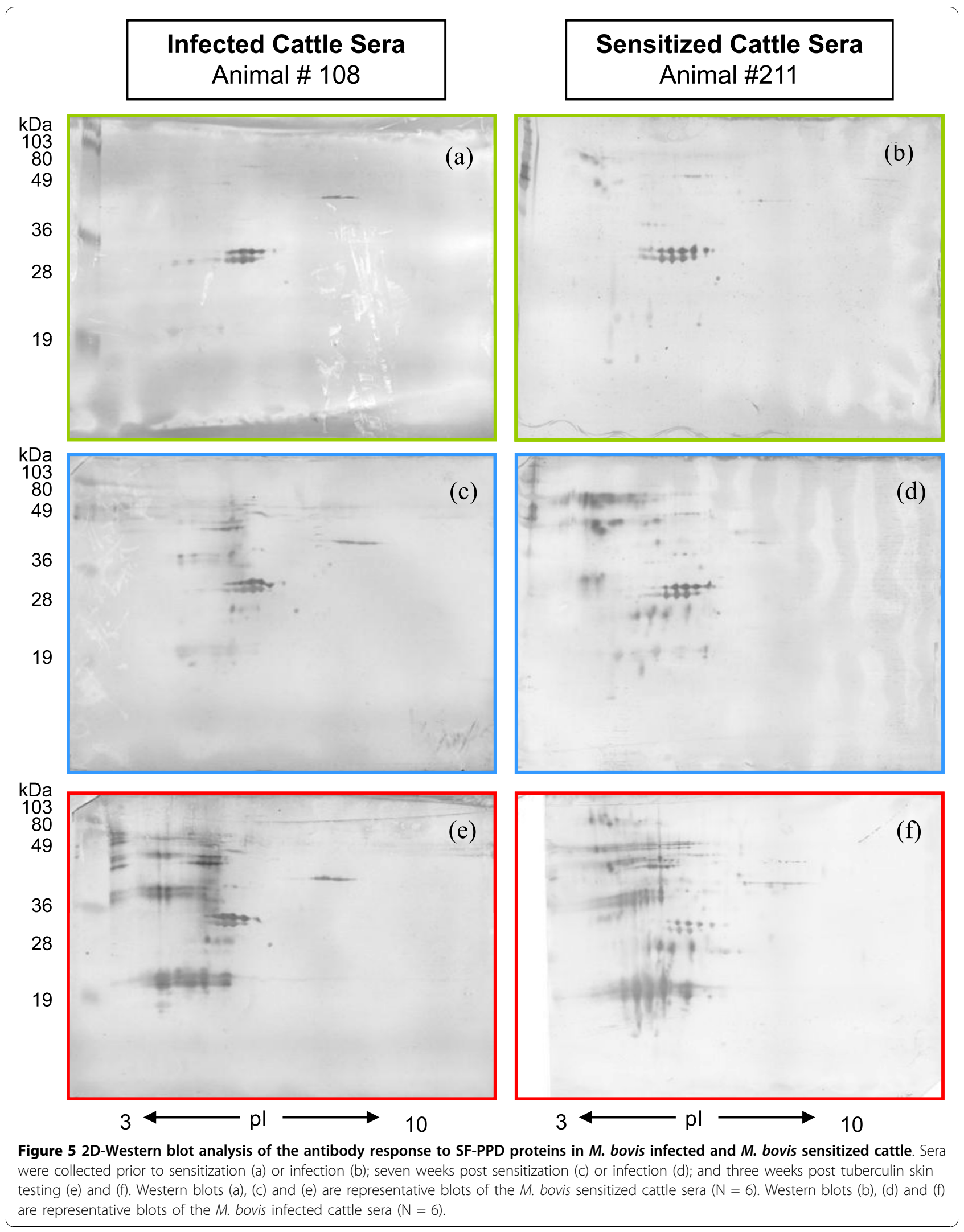


tuberculin may contain a greater number of whole tuberuclo-proteins than previously believed $[6,9,29,30]$. The protein group that appeared to have the greatest resilience to the effects of autoclaving was the MPB70 and MPB83 protein group. This result is in accordance with previous studies involving autoclaved $M$. bovis culture filtrate [9], where the heat stability of the distinct but highly homologous MPB70 and MPB83 proteins was attributed to the presence of identical 133 amino acid disulfide loops and the formation of stable aggregates in the culture fluid [31,32]. The detection of MPB32 in HK-PPD (faintly visible in Additional file 8 FIG. S8) is especially interesting as this protein was described by Nagai $e t a l$. as the most heat labile protein as compared to MPB64 and the Ag85 complex proteins [10].

The suspected presence of heat liable M. bovis CFPs in PPD tuberculin therefore stresses the importance of conformity between bulk lots of PPD tuberculin with respect to the heat kill parameters. Reduced autoclave time and/ or pressure would likely increase the concentration of whole proteins whereas increased time and pressure may reduce even the peptide fragments to non-antigenic subunits. This has been shown by Borsuk et al [33] who reported that $M$. bovis protein rV3874 was the most abundant tuberculo-protein preset in $M$. bovis PPD tuberculin produced in the UK while the same protein did not rank is the top ten most abundant tuberculoproteins found in M. bovis PPD produced in Brazil. Although both the UK and Brazilian PPDs were derived from $M$. bovis AN5, the heat kill parameters used to produce the two tuberculins differed greatly While Trevedi et al. [34] reported no significant difference between the DTH response elicited by three-week-old $M$. bovis CFPs as compared to those provoked by M. bovis PPD tuberculin in $M$. bovis infected cattle, the immunological significance of the concentration of $M$. bovis peptide fragments in PPD tuberculin remains to be elucidated.

The 2-DE gels of HK-PPD also displayed protein spots which were not observed in 2-DE of SF-PPD (Figure 3b). These HK-PPD spots were located between $10-15 \mathrm{kDa}$ and possessed approximate pIs of $6-8$. While these spots could represent additional cytoplasmic proteins released into HK-PPD through the autoclave process, we did not detect antibody recognition to these protein spots in corresponding locations using Western blot analysis (data not shown). Due to the low molecular weight of these proteins and the failure to detect these spots in 2-DE of SF-PPD, it is suspected that these spots actually represented complexes of CFPs which were reduced to peptide fragments by the autoclaving process. The immunological significance of these protein spots with respect to the tuberculin skin test response has yet to be determined.
The majority of approximately 200 distinct protein spots observed on 2-DE gels of SF-PPD were easily visualized and clearly delineated as compared to the smeared presentation of HK-PPD. Although this number falls well short of the 800 plus protein spots reported to exist in $M$. bovis culture filtrate [12], the MASS-SPEC results from this study indicated that each protein spot actually contained a mixture of multiple $M$. bovis CFPs (TABLE 1). Another potential reason for this difference in detected protein spots is that our preparation of M. bovis CFPs was obtained from 9-week-old culture supernatant in order to characterize the actual constituents that make up PPD tuberculin whereas other studies used shorter culture times for their CFP preparations [12,35]. The concentration of CFPs changes as the culture time increases, with the more stable proteins, for example MPB70/83, building up over time while others, such as MPB64 tend to degrade in the culture supernatant [14].

One-dimensional Western blots of sera from M. bovis infected or sensitized cattle onto HK-PPD revealed that the majority of antibody binding to HK-PPD was largely restricted to a $22-\mathrm{kDa}$ band. However, when the same sera were blotted to SF-PPD additional bands were visualized. The $22 \mathrm{kDa}$ band observed in blots of HK-PPD has previously been interpreted to indicate reactivity to both MPB70 and MPB83, however, the two additional weak bands with apparent weights of 40 and $66 \mathrm{kDa}$, (Figure 2 lanes 4 \& 5) may also have consisted of MPB70 and MPB83 as these proteins have previously been shown to present as dimers and trimers in Western blots of $M$. bovis CFPs [31,32]. The streaked appearance of HK-PPD Western blots is suspected to result from the HK-PPD peptide fragments which are recognized by antibodies in the $M$. bovis sensitized/ infected sera. The background effect resulting from these peptide fragments likely masked the antibody responses to small quantities of whole HK-PPD proteins. Therefore, although the antibodies in $M$. bovis sensitized sera may have recognised peptide fragments originating from a multitude of HK-PPD antigens, we were unable to ascertain from which M. bovis CFPs the peptide fragments originated.

The occurrence of antibody recognition of SF-PPD antigen in all twelve pre-infection/sensitization cattle sera was unexpected (Additional files 1, 2, 3, 4, 5 and 6, FIG. S1-S6), especially since all twelve animals tested negative on the pre-screening INF-(based in vitro test for bovine tuberculosis (results not shown). Although the pre-existing antibody level to SF-PPD antigens varied between animals, all twelve cattle recognized a similar group of SF-PPD proteins in 2D Western blot analysis which corresponded to the Ag85 complex proteins identified by MASS-SPEC analysis (Figure 3a, 
Figure 5a, b). Interestingly, this background antibody level did not appear to be correlated to animal age or to isolation of housing facility. Cattle designated for the infection trial were purchased from a single farm at four months of age and maintained within a Bio-containment Level III facility while cattle designated for the M. bovis sensitization trial were purchased from various local farms and maintained to approximately 1.5 years of age in an open air, large animal facility prior to the initiation of the study.

Ag85 complex proteins are not specific to Mycobacterium tuberculosis complex strains but are also known to be expressed by most environmental mycobacteria [36,37]. M. avium and M. bovis Ag85 complex proteins reportedly share $99 \%$ homology at the protein levels [38] and both Santema et al and Borsuk et al have identified Ag85 complex proteins in $M$. avium PPD by MASSSPEC $[33,39]$. Therefore it is not surprising that cross reactive epitopes have been shown between the respective Ag85 complexes [10]. Both Mustafa et al. and Amadori et al. $[30,40]$ credited a prior exposure to environmental mycobacteria as the basis for low levels of Ag85 complex antibody recognition in $M$. bovis negative cattle. Espitia et al. and Al-Attiyah et al. [41,42] also attributed environmental mycobacteria as a potential determinant for Ag85 complex antibody recognition in the sera of tuberculosis-free humans. Therefore, the presence of Ag85 in PPD tuberculin may contribute to the relatively low specificity of the TST. An analysis of M. avium PPD tuberculin by 2DE and 2D Western blot analysis may help elucidate other potentially cross-reactive proteins.

Additional SF-PPD antigenic determinants were detected from 2D Western blots of sera from M. bovis sensitized cattle as compared to $M$. bovis infected cattle at seven weeks post $M$. bovis infection/sensitization. Little difference was noted in the antibody response to SF-PPD antigens in sera taken at seven weeks post infection as compared to the pre-infection sera. This was in accordance with other previous $M$. bovis infection studies in which antibody response to $M$. bovis CFP antigens were initially detected between 7-10 weeks post infection $[43,44]$. One reason for the noted difference between the infected and sensitized cattle in our study may be explained by the antigenic dose. Cattle sensitized with a $20 \mathrm{mg}$ intramuscular injection of heat killed $M$. bovis cells may have been exposed to a higher dose of $M$. bovis CFPs in the first seven weeks of this study as compared to the infected cattle that received 1500 CFU of live M. bovis by intra-tracheal inoculation.

Application of the CITST resulted in a dramatic boosting of the antibody response to SF-PPD in both the $M$. bovis infected and $M$. bovis sensitized cattle. While several other studies have indicated a similar boosting effect caused by tuberculin for both the humoral $[1,9,20,44,45]$ and cellular $[1,19,46]$ immune responses of $M$. bovis infected cattle, the precise mechanisms by which this occurs remains to be determined [35]. Harboe et al. [43] originally hypothesized that the marked increase in antibody response following skin testing in cattle was principally due to the presence of native MPB70 in M. bovis PPD tuberculin. Harboe et al. (1990) further postulated that a similar antibody boosting effect following a TST was typically not observed in human tuberculosis patients due to the minimal amount of MPB70 present in M. tuberculosis PPD tuberculin [43]. This is contradictory to our results that indicated a general increase in antibody response to several SF-PPD proteins. The disparity of results between Harboe et al. [43] and the findings in this study may be explained, in part, by the different methods used to analyse the sera. Our use of 2D Western blots presumably provided an increased separation and sensitivity of the $M$. bovis CFPs as compared to the one dimensional Westerns performed by Harboe et al. [43]. The increased separation of $M$. bovis CFPs may have therefore permitted a more precise analysis of the antigen recognition by serum antibodies. Our observations do concur with the results of an $M$. bovis infection study by Lyashchenko et al. which indicated that the application of a TST induced an antibody boost to several other $M$. bovis CFPs proteins including MPB64 [1]. While the mechanism of this boosting effect remains to be completely characterized, it is likely that peripheral B-cells are stimulated to produce antibodies to predominantly linear epitopes of the soluble PPD tuberculin proteins following cognate interactions with $\mathrm{T}$-cells.

We did not observe significant antibody responses to the SF-PPD proteins of molecular weights less than 20 $\mathrm{kDa}$ in $M$. bovis infected or sensitized cattle sera. This result was in contradiction to findings by other researchers who showed that both ESAT- 6 and CFP10 (which are reported to resolve at approximately 8 and $12 \mathrm{kDa}$ respectively in 2-DE analyses of mycobacterial CFPs [17], have previously been shown to elicit an antibody boosting effect in $M$. bovis infected cattle following skin testing $[1,9,20,44]$. Since ESAT-6 is known to be secreted in the early stages of $M$. bovis infection [47-50], it is suspected that the $M$. bovis infected cattle would have elicited an antibody response to ESAT-6 and/or CFP10. However, our Western blot analysis was not able to detect significant antibody response at 8 and/or $12 \mathrm{kDa}$. Of note, ESAT- 6 and CFP-10 proteins were detected in 2-DE of SF-PPD by MASS-SPEC analysis at $22 \mathrm{kDa}, 4.2$ \& $4.7 \mathrm{pI}$ respectively (Additional files 1, 2, 3, 4, 5 and 6, FIG. S1-S6). While this indicates that ESAT6 and CFP-10 are present in SF-PPD, it does not allude to their respective concentrations. In addition the 9 
week long propagation of $M$. bovis cultures likely does not favour maximum yield of ESAT- 6 and CFP-10 as the proteins with increased structural integrity, for example MPB70/83, are more suited to persist in the culture fluid following secretion. Furthermore, the dialysis and concentration of $M$. bovis culture filtrate at 10 $\mathrm{kDa}$ may have resulted in the partial loss of these two proteins from both HK-PPD and SF-PPD preparations. Therefore, the detection of ESAT- 6 and CFP-10 in SF-PPD at $22 \mathrm{kDa}$ is likely an example of the sensitivity of MASS-SPEC as opposed to the key location for these proteins.

The use of a more specific/sensitive technique such as multi-antigen print immunoassay (MAPIA) which employs recombinant mycobacterial proteins, may be required to detect serum antibodies to ESAT- 6 and CFP-10 as has been used previously $[1,19,20,46]$. While our Western blotting technique involved denaturation of protein by SDS and 2ME, thereby restricting our analysis to linear epitopes, MAPIA and select (-INF based diagnostic tests use recombinant proteins in a native conformation. Therefore, serum antibodies specific for conformational epitopes may recognise the native conformation of the antigens used in MAPIA that could thereby alter the antibody recognition for a given tuberculo-protein. While the presence and quantity of both ESAT- 6 and CFP10 in SF-PPD may be determined by future studies using monoclonal antibodies specific for the respective proteins or another more sensitive method, the existence of an antibody response to either ESAT-6 or CFP10 from M. bovis infected/sensitized cattle cannot be concluded from this study.

\section{Conclusions}

M. bovis PPD tuberculin has been used for more than a century to control one of the most devastating bacterial diseases of all times however the actual antigenic constituents of $M$. bovis PPD tuberculin and the immunological events initiated by its use have yet to be fully understood. The main purpose of this study was to characterize the antigenic constituents of $M$. bovis CFPs and to compare the antibody response of $M$. bovis infected cattle to that of cattle artificially sensitized to $M$. bovis by 2D Western blot analysis. Our 2-DE analysis of HK-PPD revealed that PPD tuberculin likely consists of a multitude of whole $M$. bovis proteins in addition to peptide fragments originating from $M$. bovis CFPs.

We concluded that it is the heat sterilization of the $M$. bovis CFPs which caused the severe structural changes and protein fragmentation observed in the HK-PPD M. bovis proteins. This work also suggested that $M$. bovis infected cattle and cattle artificially sensitized to $M$. bovis with an injection of heat-killed cells exhibited similar antibody response to the $M$. bovis antigens under study. While this study compares the humoral immune response of $M$. bovis infected and $M$. bovis sensitized cattle, future comparisons of their cellular immune responses may lead an $M$. bovis sensitization method which would provide a consistent immune response similar to that of an experimentally $M$. bovis infected animals. The development of an accurate, noninfectious bovine tuberculosis model would reduce the complexity and bio-containment risks associated with live M. bovis studies.

\section{Additional material}

Additional file 1: Fig. S1. Western blot analysis of the antibody response to SF-PPD proteins in cattle prior to $M$. bovis sensitization.

Additional file 2: Fig. S2. Western blot analysis of the antibody response to SF-PPD proteins in cattle at seven weeks post $M$. bovis sensitization.

Additional file 3: Fig. S3. Western blot analysis of the antibody response to SF-PPD proteins in $M$. bovis sensitized cattle post CITST.

Additional file 4: Fig. S4. Western blot analysis of the antibody response to SF-PPD proteins in cattle prior to $M$. bovis infection.

Additional file 5: Fig. S5. Western blot analysis of the antibody response to SF-PPD proteins in cattle at seven weeks post M. bovis infection.

Additional file 6: Fig. S6. Western blot analysis of the antibody response to SF-PPD proteins in M. bovis infected cattle post CITST.

Additional file 7: Fig. S7. Western blot analysis of the antibody response to SF-PPD proteins in negative control cattle \#9246. (not sensitized to, or infected with $M$. bovis.). (a) Pre-injection of mineral oil/ lanoline; (b) seven weeks post mineral oil/lanoline injection; (c) Post CITST.

Additional file 8: Fig. S8. Silver stained 2-DE analysis of HK-PPD depicting MPB32. $50 \mu \mathrm{g}$ of HK-PPD was loaded onto $17 \mathrm{~cm}$ pre-cast acrylamide strip with an immobilized $\mathrm{pH}$ range of 3-10 (ReadyStrip ${ }^{\mathrm{TM}}$; BioRad). Following isoelectric focussing, the acrylamide strip was loaded into a vertical $12 \%$ polyacrylamide gel with molecular weight standard (Silver Stain SDS-PAGE Standards, Low Range, Bio-Rad).

\section{Acknowledgements}

The authors would like to thank Bridgette Sognier, Barb du Pree and Jennifer Hazelwood for their technical assistance; Dr. Scott, Dr. Pelchat, Dr Brooks and Dr. Lin for their council and technical expertise. This work was funded by the Canadian Food Inspection Agency and was performed as a partial fulfilment of BR's MSc degree while he was on education leave.

\section{Author details}

${ }^{1}$ Canadian Food Inspection Agency, Mycobacterial Diseases, Ottawa, K2H 8P9, Canada. ${ }^{2}$ Department of Biochemistry, Microbiology and Immunology, Faculty of Medicine, University of Ottawa, Ottawa, K1H 8M5, Canada.

\section{Authors' contributions}

BR performed all of the laboratory work as a partial fulfillment of his MSC degree at the University of Ottawa. BR wrote the manuscript and the two senior authors (LF and NS) corrected and approved the manuscript. All authors participated in the conception of the study and participated in its design and coordination. All authors read and approved the final manuscript. 
Received: 4 March 2010 Accepted: 9 November 2010

Published: 9 November 2010

\section{References}

1. Lyashchenko K, Whelan AO, Greenwald R, Pollock JM, Andersen P, Hewinson RG, et al: Association of tuberculin-boosted antibody responses with pathology and cell-mediated immunity in cattle vaccinated with Mycobacterium bovis BCG and infected with M. bovis. Infect Immun 2004, 72:2462-2467.

2. Landi S: Production and standardization of tuberculin (a brief history). Indian J Chest Dis Allied Sci 1982, 24:78-87.

3. Nagai S, Matsumoto J, Nagasuga T: Specific skin-reactive protein from culture filtrate of Mycobacterium bovis BCG. Infect Immun 1981, 31:1152-1160.

4. Affronti L, Porrello V, Gupta S: Trace elements incorporated into the culture medium of Mycobacterium tuberculosis promiote the presence of tuberculoprotein $\mathrm{C}$ in the preparation of purified protein derivatives. Microbios 1990, 63:101-107.

5. Seibert FB: The isolation of three different proteins and two polysacchrides from tuberculin by alcohol fractionation. Their chemical and biological properties. Am Rev Tuberc 1949, 59:86-101.

6. Augier J, Augier-Gibory S, Romain F, Versmisse E: Search of a standard for the titration of antigen L. Dev Biol Stand 1986, 58(Pt B):495-501.

7. Chase MW: Multiple disseminated granulomata in sensitized guinea pigs. Ryumachi 1975, 15:389-390.

8. Landi S, Held HR, Gupta KC: The multi-facets of tuberculin standardization. Dev Biol Stand 1975, 29:393-411.

9. Harboe M: Antigens of PPD, old tuberculin, and autoclaved Mycobacterium bovis BCG studied by crossed immunoelectrophoresis. Am Rev Respir Dis 1981, 124:80-87.

10. Nagai $S$, Wiker HG, Harboe M, Kinomoto M: Isolation and partial characterization of major protein antigens in the culture fluid of Mycobacterium tuberculosis. Infect Immun 1991, 59:372-382

11. Fifis T, Costopoulos C, Radford A, Bacic A, Wood PR: Purification and Characterization of Major Anitgens from a Mycobacterium bovis Culture Filtrate. Infect Immun 1991, 59:800-807.

12. Mattow J, Schaible UE, Schmidt F, Hagens K, Siejak F, Brestrich G, et al: Comparative proteome analysis of culture supernatant proteins from virulent Mycobacterium tuberculosis $\mathrm{H} 37 \mathrm{Rv}$ and attenuated $\mathrm{M}$. bovis BCG Copenhagen. Electrophoresis 2003, 24:3405-3420.

13. Sonnenberg MG, Belisle JT: Definition of Mycobacterium tuberculosis culture filtrate proteins by two-dimensional polyacrylamide gel electrophoresis, $\mathrm{N}$-terminal amino acid sequencing, and electrospray mass spectrometry. Infect Immun 1997, 65:4515-4524.

14. Andersen P, Askgaard D, Ljungqvist L, Bennedsen J, Heron I: Proteins released from Mycobacterium tuberculosis during growth. Infect Immun 1991, 59:1905-1910.

15. Jungblut PR, Schaible UE, Mollenkopf HJ, Zimny-Arndt U, Raupach B, Mattow J, et al: Comparative proteome analysis of Mycobacterium tuberculosis and Mycobacterium bovis BCG strains: towards functional genomics of microbial pathogens. Mol Microbio/ 1999, 33:1103-1117.

16. Malen H, Softeland T, Wiker HG: Antigen analysis of Mycobacterium tuberculosis H37Rv culture filtrate proteins. Scand J Immunol 2008 67:245-252.

17. Rosenkrands I, Weldingh K, Jacobsen S, Hansen CV, Florio W, Gianetri I, et al: Mapping and identification of Mycobacterium tuberculosis proteins by two-dimensional gel electrophoresis, microsequencing and immunodetection. Electrophoresis 2000, 21:935-948.

18. World Organization for Animal Health: Bovine tuberculosis. Manual of Diagnostic Tests and Vaccines for Terrestrial Animals Paris, France.: Office International des Episooties; 2004

19. Palmer MV, Waters WR, Thacker TC, Greenwald R, Esfandiari J, Lyashchenko KP: Effects of different tuberculin skin-testing regimens on gamma interferon and antibody responses in cattle experimentally infected with Mycobacterium bovis. Clin Vaccine Immunol 2006, 13:387-394.

20. Waters WR, Palmer MV, Thacker TC, Payeur JB, Harris NB, Minion FC, et al: Immune responses to defined antigens of Mycobacterium bovis in cattle experimentally infected with Mycobacterium kansasii. Clin Vaccine Immunol 2006, 13:611-619.
21. Lowry OH, Rosebrough NJ, Farr AL, Randall RJ: Protein measurement with the Folin phenol reagent. J Biol Chem 1951, 193:265-275.

22. Laemmli UK: Cleavage of structural proteins during the assembly of the head of bacteriophage T4. Nature 1970, 227:680-685.

23. Shevchenko A, Wilm M, Vorm O, Mann M: Mass spectrometric sequencing of proteins silver-stained polyacrylamide gels. Anal Chem 1996, 68:850-858.

24. Towbin H, Staehelin T, Gordon J: Electrophoretic transfer of proteins from polyacrylamide gels to nitrocellulose sheets: proceedure and some applications. Proc Natl Acad Sci 1979, 76:4350-4354.

25. Harboe M, Nagai S, Patarroyo ME, Torres ML, Ramirez C, Cruz N: Properties of proteins MPB64, MPB70, and MPB80 of Mycobacterium bovis BCG. Infect Immun 1986, 52:293-302.

26. Ohara N, Ohara-Wada N, Kitaura H, Nishiyama T, Matsumoto S, Yamada T: Analysis of the genes encoding the antigen 85 complex and MPT51 from Mycobacterium avium. Infect Immun 1997, 65:3680-3685.

27. Weldingh K, Rosenkrands I, Jacobsen S, Rasmussen PB, Elhay M Andersen P: Two-dimensional electrophoresis for analysis of Mycobacterium tuberculosis culture filtrate and purification and characterization of six novel proteins. Infect Immun 1998, 66:3492-3500.

28. Gupta KC, Landi S: Isolation of tuberculin peptides from tuberculin purified protein derivative (PPD). Can J Microbiol 1978, 24:1242-1249.

29. Andersen AB, Ljungqvist $L$, Haslov $K$, Bentzon MW: MPB 64 possesses 'tuberculosis-complex'-specific B- and T-cell epitopes. Scand J Immunol 1991, 34:365-372.

30. Mustafa AS, Skeiky YA, Al Attiyah R, Alderson MR, Hewinson RG, Vordermeier HM: Immunogenicity of Mycobacterium tuberculosis antigens in Mycobacterium bovis BCG-vaccinated and M. bovis-infected cattle. Infect Immun 2006, 74:4566-4572.

31. Haslov $K$, Andersen $A B$, Bentzon MW: Biological activity in sensitized guinea pigs of MPB 70, a protein specific for some strains of Mycobacterium bovis BCG. Scand J Immunol 1987, 26:445-454

32. Wiker HG, Lyashchenko KP, Aksoy AM, Lightbody KA, Pollock JM, Komissarenko SV, et al: Immunochemical characterization of the MPB70/ 80 and MPB83 proteins of Mycobacterium bovis. Infect Immun 1998 66:1445-1452.

33. Borsuk S, Newcombe J, Mendum TA, Dellagostin OA, McFadden J: Identification of proteins from tuberculin purified protein derivative (PPD) by LC-MS/MS. Tuberculosis (Edinb) 2009, 89:423-430.

34. Trivedi S, Das SK, Kumar R, Chandra R: Studies on diagnostic potential of secretory antigens of Mycobacterium bovis. Indian J Exp Biol 2006, 44:14-18.

35. Villarreal-Ramos B, Reed S, McAulay M, Prentice H, Coffey T, Charleston BC, et al: Influence of the nature of the antigen on the boosting of responses to mycobacteria in $\mathrm{M}$. bovis-BCG vaccinated cattle. Vaccine 2006, 24:6850-6858.

36. Ewer K, Cockle P, Gordon S, Mansoor H, Govaerts M, Walravens K, et al: Antigen mining with iterative genome screens identifies novel diagnostics for the Mycobacterium tuberculosis complex. Clin Vaccine Immunol 2006, 13:90-97.

37. Wiker HG, Harboe M: The Antigen 85 Complex: a Major Secretion Product of Mycobacterium tuberculosis. Microbiol Rev 1992, 56:648-661.

38. Rosseels V, Marche S, Roupie V, Govaerts M, Godfroid J, Walravens K, et al: Members of the 30- to 32-Kilodalton Mycolyl Transferase Family (Ag85) from Culture Filtrate of Mycobacterium avium subsp. paratuberculosis Are Immunodominant Th1-Type Antigens Recognized Early upon Infection in Mice and Cattle. Infect Immun 2006, 74:202-212

39. Santema W, Overdijk M, Barends J, Krijgsveld J, Rutten V, Koets A: Searching for proteins of Mycobacterium avium subspecies paratuberculosis with diagnostic potential by comparative qualitative proteomic analysis of mycobacterial tuberculins. Vet Microbiol 2009, 138:191-196.

40. Amadori M, Tameni S, Scaccaglia P, Cavirani S, Archetti IL, Giandomenico RQ: Antibody tests for identification of Mycobacterium bovis-infected bovine herds. J Clin Microbiol 1998, 36:566-568.

41. Al Attiyah R, Madi N, El Shamy AS, Wiker H, Andersen P, Mustafa A: Cytokine profiles in tuberculosis patients and healthy subjects in response to complex and single antigens of Mycobacterium tuberculosis. FEMS Immunol Med Microbiol 2006, 47:254-261.

42. Espitia C, Sciutto E, Bottasso O, Gonzalez-Amaro R, Hernandez-Pando R, Mancilla R: High antibody levels to the mycobacterial fibronectin-binding 
antigen of 30-31 kD in tuberculosis and lepromatous leprosy. Clin Vaccine Immunol 1992, 87:362-367.

43. Harboe M, Wiker HG, Duncan JR, Garcia MM, Dukes TW, Brooks BW, et al: Protein G-based enzyme-linked immunosorbent assay for anti-MPB70 antibodies in bovine tuberculosis. J Clin Microbiol 1990, 28:913-921.

44. Waters WR, Palmer MV, Thacker TC, Bannantine JP, Vordermeier HM Hewinson $\mathrm{RG}$, et al: Early antibody responses to experimental Mycobacterium bovis infection of cattle. Clin Vaccine Immunol 2006, 13:648-654.

45. Thom M, Morgan JH, Hope JC, Villarreal-Ramos B, Martin M, Howard CJ: The effect of repeated tuberculin skin testing of cattle on immune responses and disease following experimental infection with Mycobacterium bovis. Vet Immunol Immunopathol 2004, 102:399-412.

46. Lyashchenko KP, Singh M, Colangeli R, Gennaro ML: A multi-antigen print immunoassay for the development of serological diagnosis of infectious diseases. J Immunol Methods 2000, 242:91-100.

47. Brodin P, Rosenkrands I, Andersen P, Cole ST, Brosch R: ESAT-6 proteins: protective antigens and virulence factors? Trends Microbiol 2004, 12:500-508.

48. Frigui W, Bottai D, Majlessi L, Monot M, Josselin E, Brodin P, et al: Control of M. tuberculosis ESAT- 6 secretion and specific T cell recognition by PhoP. PLoS Pathog 2008, 4:e33.

49. Harboe M, Wiker HG, Lachmann PJ: Carrier effect of concanavalin A-reactive and -non-reactive material in tuberculin PPD. Scand I Immunol 1990, 32:263-271.

50. Inwald J, Hinds J, Palmer S, Dale J, Butcher PD, Hewinson RG, et al: Genomic analysis of Mycobacterium tuberculosis complex strains used for production of purified protein derivative. J Clin Microbiol 2003, 41:3929-3932.

doi:10.1186/1746-6148-6-50

Cite this article as: Rennie et al: Antibody response to a sterile filtered PPD tuberculin in M. bovis infected and M. bovis sensitized cattle. BMC Veterinary Research 2010 6:50

\section{Submit your next manuscript to BioMed Central and take full advantage of:}

- Convenient online submission

- Thorough peer review

- No space constraints or color figure charges

- Immediate publication on acceptance

- Inclusion in PubMed, CAS, Scopus and Google Scholar

- Research which is freely available for redistribution 